See discussions, stats, and author profiles for this publication at: https://www.researchgate.net/publication/338923785

\title{
Tangential intersection of branches of motion
}

Preprint in Mechanism and Machine Theory · January 2020

DOI: 10.1016/j.mechmachtheory.2019.103730

CITATION

4 authors, including:

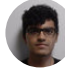

Pablo Lopez-Custodio

King's College London

19 PUBLICATIONS 84 CITATIONS

SEE PROFILE

D Xi Kang

National University of Singapore

10 PUBLICATIONS 19 CITATIONS

SEE PROFILE

Some of the authors of this publication are also working on these related projects:

Project In-Balance View project

Project Multibody Kinematics View project
READS

146

Andreas Mueller

Johannes Kepler University Linz

287 PUBLICATIONS 1,657 CITATIONS

SEE PROFILE 


\title{
Tangential intersection of branches of motion
}

\author{
P.C. López-Custodio ${ }^{\mathrm{a}, *}$, A. Müller ${ }^{\mathrm{b}}$, X. Kang ${ }^{\mathrm{c}}$, J. S. Dai ${ }^{\mathrm{a}}$ \\ ${ }^{a}$ King's College London, Strand, WC2R2LS, London, UK \\ ${ }^{b}$ Johannes Kepler University, Altenbergerstraße 69, 4040, Linz, Austria \\ ${ }^{c}$ National University of Singapore, 21 Lower Kent Ridge Rd, 119077, Singapore
}

\begin{abstract}
The branches of motion in the configuration space of a reconfigurable linkage can intersect in different ways leading to different types of singularities. In the vast majority of reported linkages whose configuration spaces contain multiple branches of motion the intersection happens transversally, allowing local methods, like the computation of its tangent cone, to identify different branches by means of their tangents. However, if these branches are of the same dimension and they intersect tangentially, it is not possible to identify them by means of the tangent cone at the singularity as the tangent spaces to the branches are the same. Although this possibility has been mentioned by a few researchers, whether linkages with this kind of tangent intersection of branches of motion exist is still an open question. In this paper, it is shown that the answer to this question is yes: A local method is proposed for the effective identification of branches of motion intersecting tangentially, and a method for the type synthesis of linkages that exhibit this particular type of singularity is presented.
\end{abstract}

Keywords: Kinematics, Configuration space, Tangential intersections, Singularity, Reconfigurable mechanisms

\section{Introduction}

The study of singularities is nothing short of crucial in the analysis, design and control of mechanisms and robots. This is a topic that has been thoroughly investigated since researchers noted that the properties of linkages dramatically 5 change in certain configurations. Of particular importance are the singularities occurring in the configuration space of the mechanism [1, 2], where its smoothness is lost. Hunt [3] called these singularities uncertainty configurations and used a planar 4-bar mechanism in a flattened configuration as an example (see also [4]), pointing out that from such configuration it is possible to unfold the

\footnotetext{
* Corresponding author

Email addresses: pablo.lopez-custodio@kcl.ac.uk (P.C. López-Custodio), a.mueller@jku.at (A. Müller), xi.kang@nus.edu.sg (X. Kang), jian.dai@kcl.ac.uk (J. S. Dai)
} 
mechanism "in two essentially different ways". These two different ways are two branches of motion, in a broad term, in the configuration space of the mechanism. An example of a 4-bar mechanism with opposite links of the same length is shown in figure 1. It can be seen that from a flattened configuration $\mathbf{q}_{0}$ the mechanism can be unfolded in two different ways: a parallelogram $\left(\mathbf{q}_{2}\right)$ and an anti-parallelogram $\left(\mathbf{q}_{1}\right)$.

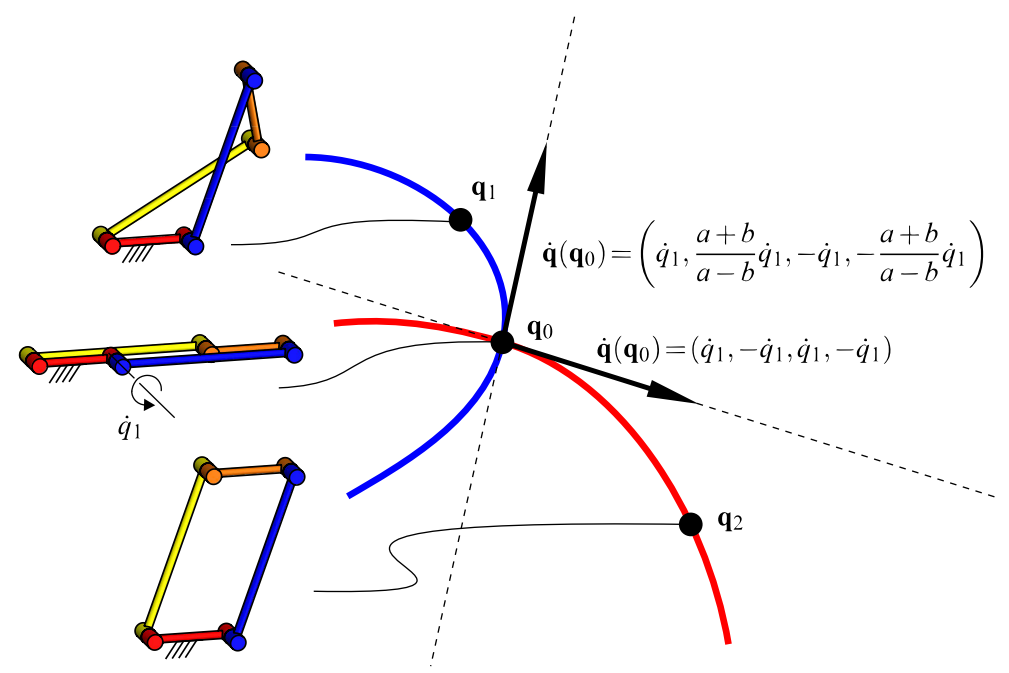

Figure 1: A 4-bar mechanism and a singularity in its configuration space.

A branch of motion, sometimes also referred to as motion mode [1, [5, 6] or motion phase 7,9$] 1$, is actually a subvariety of the real variety built by the set of feasible configurations that constitute the configuration space of the mechanism, see [5, 6, 10, 11] for examples of analysis of mechanisms using 20 algebraic geometry. Zlatanov et al [1] identified these subvarieties as regions of the configuration space and called Hunt's uncertainty configurations constraint singularities.

Most mechanisms have a configuration space that possesses non-smooth points and many include several branches of motion. Over the past twenty 25 years, several types of mechanisms with various branches of motion have been investigated. With their various features, typical types of mechanisms have been investigated: metamorphic mechanisms [9, 12 16], kinematoropic mechanisms [17 21] and discontinuously movable mechanisms [22, 23]. These are called in general reconfigurable mechanisms [24 29].

The configuration space of a mechanism is a variety and it can be modeled in particular as an analytic variety. Whitney studied the tangents to analytic varieties [30] and defined the concept of tangent cone, which, unlike the tangent space, should also describe the local aspects of the variety at its singularities.

${ }^{1}$ This term has also been applied to cases where physical limit or variable joints were used. 
The approach was firstly adapted to mechanisms theory by Lerbet [31], followed by Müller [32-34]. The concept of tangent cone was not only used in the study, but also in the design of mechanisms 35$]$.

Although singularities in the configuration space have been investigated rigorously through decades, most of this research has focused on transversal intersection of motion branches of the same and of different dimensions. In a 40 transversal intersection, the two manifolds intersecting have non-parallel tangents at the intersection point. The intersection in the configuration space of the 4-bar mechanism shown in 1 happens transversally. In figure 1, the entries of the tangent vectors $\dot{\mathbf{q}} \in \mathbb{R}^{4}$ are given by the angular velocities of the four revolute joints. At the intersection, $\mathbf{q}_{0}$, the vectors that are tangent to the 45 branch of motion related to parallelogram configurations are non-parallel to the tangent vectors to the branch of motion with anti-parallel configurations. This is depicted in figure 1, where $a$ and $b$ are the two different link lengths and $\dot{q}_{1} \in \mathbb{R}$ is the angular velocity of the input joint.

In the case of transversal intersections, the concept of tangent cone works 50 perfectly and the authors consider it a very effective tool for analyzing these singularities, as it reveals the number of motion branches intersecting as well as their dimension, i.e., the number of finite degrees of freedom of the mechanism in each branch. However, there are several other situations of intersections of branches of motion that have remained rather obscure mainly due to the lack of 55 examples of mechanisms exhibiting these types of singularities. Table 1 shows seven different cases of intersections of motion branches, it has to be warned that this table is not meant to be exhaustive.

It can be seen that cases 1 and 2 of Table 1 are common singularities with many examples being reported. In these types of singularities, the tangents to the subvarieties span different vector spaces and they can be recognized by means of the computation of the tangent cone. Cases 5, 6 and 7 involve a cusp. Only until recently, only one example of a mechanism with a cusp in the configuration space was known: Connelly and Servatius' double-Watt linkage [52]. None of the various possible definitions of tangent cone by Withney works 65 at such singularities, and it was shown in 54 that the tangent cone analysis does not work at such singularity since the tangents are not defined at the cusp. Therefore the notion of tangent cone, which was already applied by Lerbet 31], was made precise by calling it the kinematic tangent cone, denoted with $C_{\mathbf{q}}^{\mathrm{K}} V$, defined as the tangents to smooth curves through a point $\mathbf{q} \in V$ [55].

In this regard, López-Custodio et al 53] presented a method for designing this kind of mechanisms. This method also led to the investigation of two unexplored, more complex cases of intersection of branches: cases 6 and 7 in Table 1. As expected, in these two cases the tangent cone only recognizes the smooth curves passing through the singularity, failing to detect the presence 75 of the cusp. Devising an effective local analysis method that can work at this singularities is still an open problem.

Recently [51], it was found that one of the earliest examples of kinematotropic linkages, the Wunderlich mechanism [17, 50] exhibits a different type of intersection between one of its 1-DOF branches and its 2-DOF branch, these 
Table 1: Different types of intersections between motion branches. (*Examples of mechanisms exhibiting singularities of the cases 1 and 2 are very common. The references cited here are just a sample of publications focusing exclusively in this kind of mechanisms.)

\begin{tabular}{lll|l} 
Examples & $\begin{array}{l}\text { Tangent } \\
\text { cone can } \\
\text { identify } \\
\text { branches }\end{array}$ \\
reported
\end{tabular}


branches intersect tangentially, i.e. their tangents at the intersection are parallel, as in case 4 in Table 1, with the tangent cone analysis still successfully detecting branches and their dimension. However, this opened the question of whether there exist mechanisms with tangential intersections of motion branches of the same dimension, in which case the tangent cone would fail in detecting the 85 bifurcation. Piiponen et al [56] discussed this topic from the algebraic geometry point of view but so far no mechanism with such property has been reported and, no technique for the analysis of this singularity has been proposed. In this paper, we show that the answer to this question is yes. Examples of mechanisms with this type of singularity are presented for the first time and an extension

90 to the tangent cone analysis method is proposed which effectively detects these motion branches.

It is important to mention that the case of tangential intersection of motion branches is of particular interest since the mechanism can move from one motion branch to another through a $C^{n_{C}}$ curve, i.e. a curve whose first $n_{C}$ derivatives exist, while in a typical reconfiguration through a transversal intersection of motion branches the mechanism has to move through a non-differentiable $C^{0}$ curve, in which not even the first derivative exists, forcing the mechanism to stop at the singularity. This will make reconfiguration technically easier, although it is not exactly a smooth motion, which would imply a curve in $C^{\infty}$, 100 therefore the terms smooth reconfiguration and smooth kinematotropy (or regular reconfiguration and kinematotropy) used in [51, 56] are not adopted in this paper.

This paper is organized as follows: Firstly, the basic notions on the use of tangent cone analysis are presented in Section 2 In Section 3 the configuration space of the Wunderlich mechanism is used to explain the phenomenon of tangential intersection of motion branches. In Section 4 a method for detecting and analyzing tangential intersections in the configuration space is presented. Section 5 explains how to design mechanisms with this type of singularity and two examples are presented and analyzed in Section 6. Finally, conclusions are drawn in Section 7 .

\section{A brief introduction to the kinematic tangent cone analysis}

In this section, the concept of kinematic tangent cone to the configuration space of a mechanism is briefly revisited. Refer to [55] for a more in-depth read about the topic and to [57] for the computational aspects.

Let $V \subset \mathbb{V}^{n}$ be the configuration space of an $n$-joint mechanism defined as:

$$
V:=\left\{\mathbf{q} \in \mathbb{V}^{n} \mid f_{i}(\mathbf{q})=\mathbf{0}, i=1, \ldots, \gamma\right\}
$$

where $f_{i}$ is the constraint equation of loop $i$ of the mechanism comprising $\gamma$ loops. Since $f_{i}$ are analytic functions, $V$ is a real analytic variety which may comprise several subvarieties that may be of different dimension. The dimension of $V$ is, therefore, a local property which, at point $\mathbf{q}$, is equal to $\operatorname{dim}\left(T_{\mathbf{q}} V\right)$ ${ }_{120}$ if $\mathbf{q}$ is a regular point of $V$. At points where different subvarieties intersect, 
the tangent space is insufficient to determine, not only the dimension of these subvarieties, but also the topology of $V$ in the neighborhood of q. It is known that the kinematic tangent cone is a suitable tool for locally analyzing these singularities, since, unlike $T_{\mathbf{q}} V$, the tangent cone, $C_{\mathbf{q}}^{\mathrm{K}} V$, consists of the union of all the tangents to smooth curves passing through $\mathbf{q}$, rather than the linear span of them.

$C_{\mathbf{q}}^{\mathrm{K}} V$ can be computed by means of higher-order approximations using the higher-order kinematic equations:

$$
\begin{aligned}
K_{\mathbf{q}}^{i}:= & \left\{\mathbf{x}_{1} \in \mathbb{R}^{n} \mid H^{(1)}\left(\mathbf{q}, \mathbf{x}_{1}\right)=\mathbf{0}, H^{(2)}\left(\mathbf{q}, \mathbf{x}_{1}, \mathbf{x}_{2}\right)=\mathbf{0}, \ldots\right. \\
& \left.H^{(i)}\left(\mathbf{q}, \mathbf{x}_{1}, \mathbf{x}_{2}, \ldots, \mathbf{x}_{i}\right)=\mathbf{0}\right\}
\end{aligned}
$$

where, for the sake of simplicity, $\mathbf{x}_{1}:=\dot{\mathbf{q}}, \mathbf{x}_{2}:=\ddot{\mathbf{q}}, \ldots, \mathbf{x}_{i}:=\mathrm{d}^{i} \mathbf{q} / \mathrm{d} t^{i} . \quad H^{(i)}$ 130 represents the $i$-th order kinematic equation. Explicit expressions for these kinematic higher-order analyses can be found in [31, 33, 57 59]. The kinematic tangent cone is obtained as:

$$
C_{\mathbf{q}}^{\mathrm{K}} V=\lim _{m \rightarrow \infty} \bigcap_{i=1}^{m} K_{\mathbf{q}}^{i}=K_{\mathbf{q}}^{\kappa_{1}}
$$

where $\kappa_{1}$ is the order at which $\operatorname{dim}\left(K_{\mathbf{q}}^{i}\right)=\operatorname{dim}\left(K_{\mathbf{q}}^{\kappa_{1}}\right), \forall i>\kappa_{1}$. Typically, it is assumed that if $\mathbf{q}$ is the intersection of $s$ motion branches, then: $K_{\mathbf{q}}^{\kappa_{1}}=\cup_{j=1}^{s} K_{\mathbf{q}}^{\kappa_{1}(j)}$ with $K_{\mathbf{q}}^{\kappa_{1}(j)} \neq K_{\mathbf{q}}^{\kappa_{1}(i)}$ if $i \neq j$. However, in the following sections it will be pointed out that this is true only if the $s$ subvarieties intersect transversally. If there are $r$ different sets of subvarieties intersecting tangentially at $\mathbf{q}$, then $K_{\mathbf{q}}^{\kappa_{1}}$ is the union of $s-r$ vector spaces.

It is important to mention that the approach only works if the tangents to $V$ at $\mathbf{q}$ exist. In the case that such tangents are not defined, for example at cusp singularities, $C_{\mathbf{q}}^{\mathrm{K}} V$ will fail to give a local picture of the configuration space. Examples of mechanisms whose configuration space exhibit a cusp singularity were reported in [52, 53].

\section{Transversal and tangential intersections in the configuration space}

Figure 2 2 a shows a planar linkage known as the Wunderlich mechanism [50]. This planar 5-loop linkage is one of the earliest examples of kinematotropic linkages, Wohlhart [17] studied it when he introduced the term kinematotropic. Wohlhart proved that the Wunderlich mechanism can change from 1 to 2 DOFs from the singular configuration $\mathbf{q}_{0} \in V$ shown in figure 2 a.

A striking property of this singularity was pointed out in [51] where Müller and Piipponen made a local description of the configuration space of the mechanism around $\mathbf{q}_{0}$. They found out that there are two 1-dimensional branches of motion and one 2-dimensional branch of motion intersecting at the singularity 


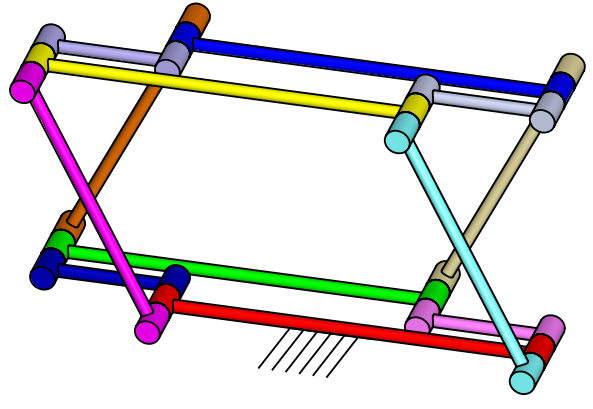

a)

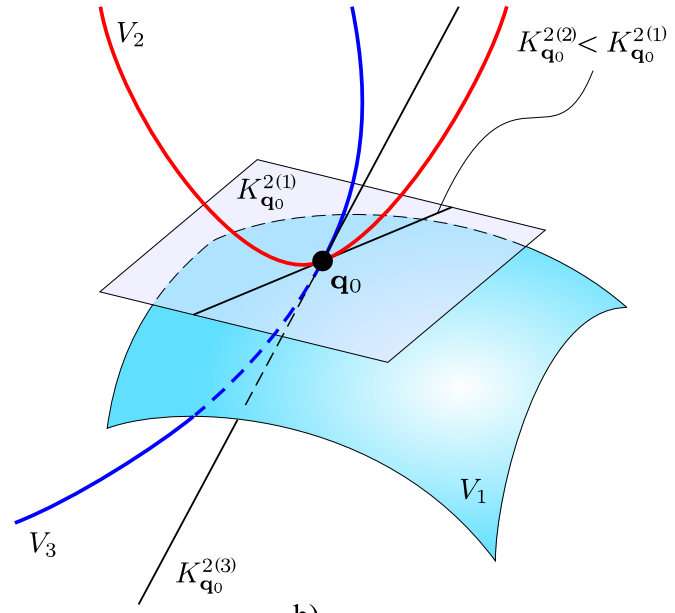

b)

Figure 2: a) The Wunderlich linkage and b) a schematic representation of its configuration space around $\mathbf{q}_{0}$.

as schematically depicted in figure2 $\mathbf{b}$. The kinematic tangent cone to $V$ at $\mathbf{q}_{0}$ is the union of the three tangent spaces to the three subvarieties intersecting at $\mathbf{q}_{0}$ and can be computed after a second-order approximation: $C_{\mathbf{q}_{0}}^{\mathrm{K}} V=\cup_{i=1}^{3} K_{\mathbf{q}_{0}}^{2(i)}$, where $\operatorname{dim}\left(K_{\mathbf{q}_{0}}^{2(1)}\right)=2$ and $\operatorname{dim}\left(K_{\mathbf{q}_{0}}^{2(2)}\right)=\operatorname{dim}\left(K_{\mathbf{q}_{0}}^{2(3)}\right)=1$. However, surprisingly $K_{\mathbf{q}_{0}}^{2(2)}<K_{\mathbf{q}_{0}}^{2(1)}$, which indicates that $V_{2}$ is a 1-dimensional curve that tangentially intersects the 2-dimensional surface $V_{1}$ as $T_{\mathbf{q}_{0}} V_{2}<T_{\mathbf{q}_{0}} V_{1}$. As far as $K_{\mathbf{q}_{0}}^{2(3)}$ is concerned, this vector space is not a subspace of $K_{\mathbf{q}_{0}}^{2(1)}$ and the intersection between $V_{1}$ and $V_{3}$ is transversal, like in any other reported reconfigurable mechanism. Nevertheless, the intersection between $V_{1}$ and $V_{2}$ proves that the Wunderlich mechanism is not only one of the earliest examples of kinematotropic linkages, but it is also the first example of a mechanism whose configuration space exhibits a tangential intersection between different subvarieties.

The tangent cone to $V$ at the analyzed singularity of the Wunderlich linkage effectively describes the local properties of the configuration space despite the peculiar property of $V_{1}$ and $V_{2}$ intersecting tangentially. The tangent cone reveals the tangents to the curves that lie in the subvarieties intersecting at the 170 analyzed point. Since $V_{1}$ and $V_{2}$ are of different dimension, their tangent spaces at $\mathbf{q}_{0}$ appear as different components of the tangent cone, namely, a line and a plane, the line being contained in the plane.

However, the situation is not as simple when dealing with the intersection of subvarieties of the same dimension. Without loss of generality, consider the 175 intersection of two 1-dimensional branches of motion. Figure 3a shows the typical case in which the branches intersect transversally at $\mathbf{q}_{0}$. If a $\kappa_{1}$ thorder approximation is required to compute the tangent cone to $V$ at $\mathbf{q}_{0}$, then $C_{\mathbf{q}_{0}}^{\mathrm{K}} V=K_{\mathbf{q}_{0}}^{\kappa_{1}(1)} \cup K_{\mathbf{q}_{0}}^{\kappa_{1}(2)}$, where $K_{\mathbf{q}_{0}}^{\kappa_{1}(1)}$ and $K_{\mathbf{q}_{0}}^{\kappa_{1}(2)}$ are two non-parallel lines, 
and $C_{\mathbf{q}_{0}}^{\mathrm{K}} V$ describes perfectly the local structure of $V$ around $\mathbf{q}_{0}$. In the case of intersections of higher-dimensional subvarieties, $C_{\mathbf{q}_{0}}^{\mathrm{K}} V$ is the union of two different vector spaces of the same dimension.

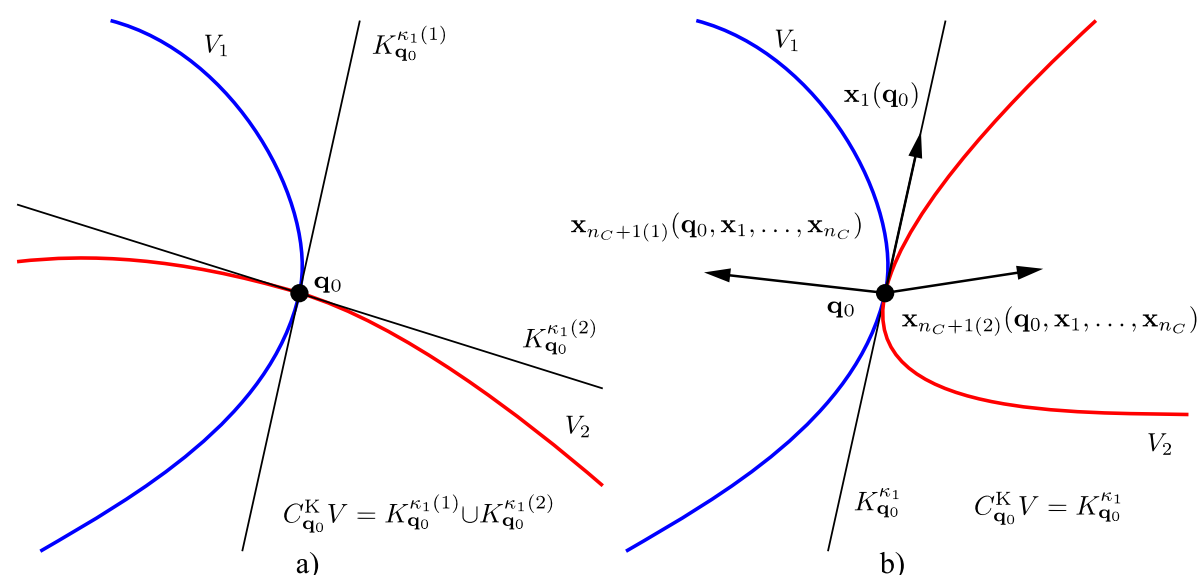

a)

b)

Figure 3: Intersection of 1-dimensional subvarieties: a) transversal case and b) tangential case.

However, if curves $V_{1}$ and $V_{2}$ intersect tangentially as shown in figure $3 \mathrm{~b}$, then $T_{\mathbf{q}_{0}} V_{1}=T_{\mathbf{q}_{0}} V_{2}$ and, hence, $K_{\mathbf{q}_{0}}^{\kappa_{1}(1)}=K_{\mathbf{q}_{0}}^{\kappa_{1}(2)}$, which means that the tangent cone, rather than being the union of two vector spaces is a sole vector space: $C_{\mathbf{q}_{0}}^{\mathrm{K}} V=$

${ }_{185} K_{\mathbf{q}_{0}}^{\kappa_{1}}$, with $K_{\mathbf{q}_{0}}^{\kappa_{1}}:=K_{\mathbf{q}_{0}}^{\kappa_{1}(i)}, i=1,2$. This means that $C_{\mathbf{q}_{0}}^{\mathrm{K}} V$ is not giving us enough information as it fails to indicate that there are two branches intersection at the analysis point, it rather suggests that there is only one tangent space to $V$ at such point, which could lead to the incorrect conclusion that there is no branching at $\mathbf{q}_{0}$. It is also important to mention that although this is a c-space 190 singularity and, as such, the rank of the Jacobian matrix will drop at such configuration, this rank decrement is a necessary but not sufficient condition for having a bifurcation in $V$ and, therefore, having a rank decrement and a tangent cone that is only one vector space is not sufficient to prove a tangent bifurcation. In [60] an example of a Goldberg 6R linkage in a configuration with a rank decrement but no bifurcation in the c-space was found.

In order to find out whether $\mathbf{q}_{0}$ is a tangent intersection further analysis is required. As shown in figure $3 \mathrm{~b}$, all vectors $a \mathbf{x}_{1}\left(\mathbf{q}_{0}\right), a \in \mathbb{R}$, which are joint velocity vectors at $\mathbf{q}_{0}$, are parallel and lie in $C_{\mathbf{q}_{0}}^{\mathrm{K}} V$, which is spanned by such vectors as $\left\{a \mathbf{x}_{1}: a \in \mathbb{R}\right\}$. However, it is possible that the joint 200 acceleration vectors $\mathbf{x}_{2}\left(\mathbf{q}_{0}, \mathbf{x}_{1}\right)$, are no longer parallel. If these vectors are still parallel they are again not useful in detecting the two branches intersecting at $\mathbf{q}_{0}$. There must exist some number $n_{C} \in \mathbb{Z}$ called order of contact, for which the $\left(n_{C}+1\right)$ th order derivatives $\mathbf{x}_{\left(n_{C}+1\right)}\left(\mathbf{q}_{0}, \mathbf{x}_{1}, \ldots, \mathbf{x}_{\left(n_{C}\right)}\right)$ span two different vector spaces allowing the identification of both branches. It is then said that all tangential intersections exhibit at least a 1st-order contact. It can be seen 
that, with this approach, the higher-order analysis is the only required tool to prove a tangent intersection. In the following section, a formal methodology that allows identifying any kind of intersection is presented.

\section{Identification of tangential intersections of branches of motion of the same dimension}

A tangential intersection at a configuration $\mathbf{q} \in V$ is characterized by the fact that there are smooth curves through $\mathbf{q}$ having the same tangents. If the intersecting branches are of the same dimension, the first-order aspect, and hence the kinematic tangent cone (any other definition of tangent cone [55]) is

215 insufficient to identify such intersection. However, the solution set of higherorder constraints can be used to this end.

The solution set of the $i$ th-order constraints is

$$
\begin{aligned}
\bar{K}_{\mathbf{q}}^{i}:=\left\{\left(\mathbf{x}_{1}, \mathbf{x}_{2}, \ldots, \mathbf{x}_{i}\right) \in \mathbb{R}^{i \times n}:\right. & H^{(1)}\left(\mathbf{q}, \mathbf{x}_{1}\right)=\mathbf{0}, \\
& H^{(2)}\left(\mathbf{q}, \mathbf{x}_{1}, \mathbf{x}_{2}\right)=\mathbf{0}, \\
& H^{(3)}\left(\mathbf{q}, \mathbf{x}_{1}, \mathbf{x}_{2}, \mathbf{x}_{3}\right)=\mathbf{0}, \\
& \\
& \left.H^{(i)}\left(\mathbf{q}, \mathbf{x}_{1}, \mathbf{x}_{2}, \mathbf{x}_{3}, \ldots, \mathbf{x}_{i}\right)=\mathbf{0}\right\} .
\end{aligned}
$$

Denote with $\pi_{k}: \mathbb{R}^{i \times n} \rightarrow \mathbb{R}^{n}$ the projection to the $k$ th factor of $\bar{K}_{\mathbf{q}}^{i}$ according to

$$
\pi_{k}\left(\bar{K}_{\mathbf{q}}^{i}\right):=\left\{\mathbf{x}_{k} \in \mathbb{R}^{n} \mid\left(\mathbf{x}_{1}, \ldots, \mathbf{x}_{k}, \ldots, \mathbf{x}_{i}\right) \in \bar{K}_{\mathbf{q}}^{i}\right\} .
$$

The $i$ th-order cone (1) is then given by

$$
K_{\mathbf{q}}^{i}=\pi_{1}\left(\bar{K}_{\mathbf{q}}^{i}\right)
$$

At a singularity, $\bar{K}_{\mathbf{q}}^{i}$ splits into the union of $s_{i}$ solution sets

$$
\bar{K}_{\mathbf{q}}^{i}=\bar{K}_{\mathbf{q}}^{i(1)} \cup \ldots \cup \bar{K}_{\mathbf{q}}^{i\left(s_{i}\right)} .
$$

where $i>n_{C}$ and $n_{C}$ is the order of contact between the motion branches intersecting tangentially at $\mathbf{q}$.

Different motion branches meeting at $\mathbf{q} \in V$ can be separated by investigating higher-order motions, which is not taken into consideration in (11). At a tangential intersection there are different higher-order motions, according to the motion branch, which have the same tangents.

Lemma 1. A point $\mathbf{q} \in V$ is a tangential intersection if and only if $\pi_{1}\left(\bar{K}_{\mathbf{q}}^{i(j)}\right) \leq$ $\pi_{1}\left(\bar{K}_{\mathbf{q}}^{i(l)}\right)$ and $\pi_{i}\left(\bar{K}_{\mathbf{q}}^{i(j)}\right) \neq \pi_{i}\left(\bar{K}_{\mathbf{q}}^{i(l)}\right), j \neq l$ for some $i>n_{C}$. If the branches 230 of motion are of the same dimension the equal symbol is fulfilled in the first condition. 
The necessary order $i$ may be different from $\kappa_{1}$ in Eq. (2). Notice that $\mathbf{q}$ may additionally be a singularity due to non-smoothness of $V$ (e.g. cusp), i.e. when $\mathbf{q}$ is a boundary point of a motion branch.

Similarly to $\operatorname{dim}\left(K_{\mathbf{q}_{0}}^{i}\right), \operatorname{dim}\left(\pi_{i}\left(\bar{K}_{\mathbf{q}}^{k}\right)\right), k>i$, decreases as $k$ increases, until at order $\kappa_{i}$ one has $\operatorname{dim}\left(\pi_{i}\left(\bar{K}_{\mathbf{q}}^{\kappa_{i}}\right)\right)=i \operatorname{dim}\left(K_{\mathbf{q}}^{\kappa_{1}}\right)$, which will no longer decrease and it can be concluded that the set of $\mathbf{x}_{i}$ vectors has been computed.

\section{Design of 1-DOF linkages with a tangential intersection in the configuration space}

A mechanism with two motion branches intersecting tangentially can be designed taking a linkage that is known to have at least two motion branches and then forcing the two branches to have the same joint velocities at their intersection configuration. Following this idea, a procedure for the design of these mechanisms is presented for the 1-DOF case.

Consider a 1-DOF mechanism that is known to have two branches of motion, $\alpha$ and $\beta$, which are intersecting at a singular configuration $\mathbf{q}_{0} \in V_{\alpha} \cap V_{\beta}$. One way to make sure the joint velocities, $\mathbf{x}_{1}$, are the same in both branches at $\mathbf{q}_{0}$ is to have one joint that is idle in branch $\alpha$, which implies that its velocity is 0 not only at $\mathbf{q}_{0}$ but at any point in $V_{\alpha}$, while in branch $\beta$ the same joint is in 250 a stationary configuration [3] at $\mathbf{q}_{0}$, i.e., its velocity is 0 in that configuration but it is different from 0 in the neighborhood of $\mathbf{q}_{0}$, it is also usual to say that the joint is in a dead-point or transitorily inactive [3]. A simple way to design a mechanism with such a property in one of its joins is now explained. We start by taking an overconstrained $6 \mathrm{R}$ linkage in which the axes of four of its joins,

${ }_{255} \mathbf{S}_{\Pi 1}, \ldots, \mathbf{S}_{\Pi 4}$, lie on a plane $\Pi$ in the configuration $\mathbf{q}_{0}$. The other two joint axes, $\mathbf{S}_{\Lambda 1}$ and $\mathbf{S}_{\Lambda 2}$, are perpendicular to $\Pi$ at $\mathbf{q}_{0}$. This arrangement is shown in figure 4. It is also required that in this configuration the $6 \mathrm{R}$ mechanism is not in a singular configuration.

Define $\mathscr{S}_{\Pi}:=\operatorname{span}\left(\mathbf{S}_{\Pi 1}\left(\mathbf{q}_{0}\right), \ldots, \mathbf{S}_{\Pi 4}\left(\mathbf{q}_{0}\right)\right)$ and $\mathscr{S}_{6 \mathrm{R}}:=\operatorname{span}\left(\mathscr{S}_{\Pi}, \mathbf{S}_{\Lambda 1}\left(\mathbf{q}_{0}\right)\right.$, ${ }_{260} \mathbf{S}_{\Lambda 2}\left(\mathbf{q}_{0}\right)$ ), clearly $\left\{\mathbf{S}_{\Lambda 1}\left(\mathbf{q}_{0}\right), \mathbf{S}_{\Lambda 2}\left(\mathbf{q}_{0}\right)\right\} \nsubseteq \mathscr{S}_{\Pi}$, and since the $6 \mathrm{R}$ mechanism is not in a singular configuration and $\mathscr{S}_{\Pi}$ is a 3 -system, $\operatorname{dim}\left(\mathscr{S}_{6 \mathrm{R}}\right)=5$. This implies that, in the equation $\sum_{i=1}^{4} \dot{q}_{\Pi i} \mathbf{S}_{\Pi i}+\sum_{i=1}^{2} \dot{q}_{\Lambda i} \mathbf{S}_{\Lambda i}=\mathbf{0}$, the solution includes $\dot{q}_{\Lambda 1}\left(\mathbf{q}_{0}\right)=\dot{q}_{\Lambda 2}\left(\mathbf{q}_{0}\right)=0$, therefore, these joins are transitorily inactive. This conclusion can be taken as a corollary to Theorem 2 of Section 2.2 in 3 ].

In order to design a reconfigurable mechanism based on this $6 \mathrm{R}$ overconstrained mechanism, let us insert a seventh $\mathrm{R}$ joint with axis $\mathbf{S}_{7 \mathrm{R}}$. This $7 \mathrm{R}$ mechanism has a branch of motion $\alpha$ in which the joint with axis $\mathbf{S}_{7 \mathrm{R}}$ is idle if $\mathbf{S}_{7 \mathrm{R}}$ is different to all the axes in the $6 \mathrm{R}$ mechanism, which means the $7 \mathrm{R}$ mechanism is still 1-DOF and follows the same motion as it was the $6 \mathrm{R}$ linkage.

270 Another branch of motion, $\beta$, appears as a non-overconstrained motion with all seven $\mathrm{R}$ joints being active, since this is a non-overconstrained motion the branch is also 1-DOF.

In order to have $\dot{q}_{7 \mathrm{R}}\left(\mathbf{q}_{0}\right)=0$ in branch $\beta$ so that both branches intersect tangentially, it is necessary to have $\mathbf{S}_{7 \mathrm{R}}\left(\mathbf{q}_{0}\right)$ parallel to $\mathbf{S}_{\Lambda 1}\left(\mathbf{q}_{0}\right)$ and $\mathbf{S}_{\Lambda 2}\left(\mathbf{q}_{0}\right)$. 


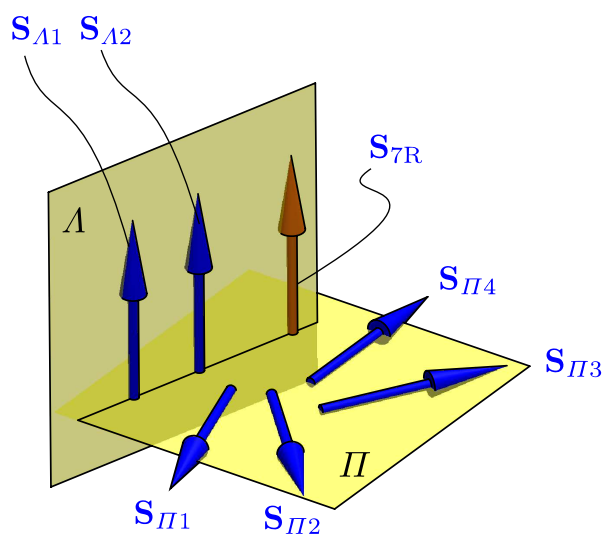

Figure 4: Joint axes of a 1-DOF mechanism with tangential intersections in the configuration space.

275 It is also required that the $7 \mathrm{R}$ mechanism is in a singular configuration as subvarieties $V_{\alpha}$ and $V_{\beta}$ are intersecting at $\mathbf{q}_{0}$. Therefore we need $\mathbf{S}_{7 \mathrm{R}}\left(\mathbf{q}_{0}\right) \in$ $\mathscr{S}_{6 \mathrm{R}}$, this is fulfilled by placing $\mathbf{S}_{7 \mathrm{R}}\left(\mathbf{q}_{0}\right)$ in $\Lambda$, the plane containing $\mathbf{S}_{\Lambda 1}\left(\mathbf{q}_{0}\right)$ and $\mathbf{S}_{\Lambda 2}\left(\mathbf{q}_{0}\right)$ (see figure 4).

For this $7 \mathrm{R}$ mechanism in configuration $\mathbf{q}_{0}$, it can be seen that $\operatorname{dim}\left(\operatorname{span}\left(\mathscr{S}_{6 \mathrm{R}}\right.\right.$, $\left.\left.\mathbf{S}_{7 \mathrm{R}}\left(\mathbf{q}_{0}\right)\right)\right)=5$, therefore $\mathbf{q}_{0}$ is singular as we know both branches of motion are 1-dimensional. In addition, due to the geometry of the joint axes belonging to perpendicular planes, $\dot{q}_{7 \mathrm{R}}\left(\mathbf{q}_{0}\right), \dot{q}_{\Lambda 1}\left(\mathbf{q}_{0}\right)$ and $\dot{q}_{\Lambda 2}\left(\mathbf{q}_{0}\right)$ cannot be written in terms of any velocity of the joints whose axes lie on $\Pi$, therefore, $\dot{q}_{7 \mathrm{R}}\left(\mathbf{q}_{0}\right)=\dot{q}_{\Lambda 1}\left(\mathbf{q}_{0}\right)=\dot{q}_{\Lambda 2}\left(\mathbf{q}_{0}\right)=0$.

285 It is important to mention that the above procedure makes sure the $7 \mathrm{R}$ linkage is in a singular configuration and the angular velocities of the joints with axes lying on plane $\Lambda$ are 0 . However, there is still a possibility of obtaining a rather strange situation in which the mechanism cannot work as a non-overconstrained $7 \mathrm{R}$ mechanism without inactive joints. In this situation, there is only one branch of motion passing through $\mathbf{q}_{0}$ in which the inserted seventh joint is always inactive, however the dimension of the screw system spanned by all 7 joints is 5 only at $\mathbf{q}_{0}$. This kind of singularity in which there is no bifurcation is rather strange, see example 7.4 of [60] for a case of this type. Nevertheless, this situation can be identified when using the analysis method 295 described in Section 4, not only does the method reveal the branching at the solution set of the order higher than the order of contact, it also shows that the time derivative of this order of the joint variable $q_{7 \mathrm{R}}$ is different to 0 , indicating that the joint is not inactive.

\section{Examples}

300

In this section two examples of reconfigurable mechanisms with two branches of motion intersecting tangentially are presented. Both examples are obtained 
using the method discussed in Section 5. The tangential intersection is identified using the concepts discussed in Section 4. See 31, 33, 57 59] for more detailed examples of the computation of the solutions to the higher-order kinematic analyses.

\subsection{Case 1: A line-symmetric-based 7R mechanism}

Figure 5 5 shows a $7 \mathrm{R}$ mechanism in which joints with axes $\mathbf{S}_{\Pi 1}, \mathbf{S}_{\Pi 2} \mathbf{S}_{\Lambda 1}$, $\mathbf{S}_{\Pi 3}, \mathbf{S}_{\Pi 4}$ and $\mathbf{S}_{\Lambda 2}$ constitute a Bricard line-symmetric $6 \mathrm{R}$ linkage [61, 62]. As shown in figure $5 \mathrm{~b}$, in the configuration $\mathbf{q}_{0} \in V$, axes $\mathbf{S}_{\Pi 1}, \ldots, \mathbf{S}_{\Pi 4}$ lie on plane $310 \Pi$, while axes $\mathbf{S}_{\Lambda 1}$ and $\mathbf{S}_{\Lambda 2}$ lie on $\Lambda$ and are perpendicular to $\Pi$. A seventh $\mathrm{R}$ joint is inserted between joints with axes $\mathbf{S}_{\Pi 1}$ and $\mathbf{S}_{\Lambda 2}$. At $\mathbf{q}_{0}$, the axis of the seventh joint, $\mathbf{S}_{7 \mathrm{R}}$, is parallel to $\mathbf{S}_{\Lambda 1}$ and also lies on $\Lambda$.

The screw coordinates with respect to the coordinate system with origin at $O$ shown in figure $5 \mathrm{~b}$ are the following:

$$
\begin{aligned}
& \mathbf{S}_{\Pi 1}\left(\mathbf{q}_{0}\right):=(0,-1,0 ; 0,0,0), \\
& \mathbf{S}_{\Pi 2}\left(\mathbf{q}_{0}\right):=(-1,0,0 ; 0,0,0), \\
& \mathbf{S}_{\Lambda 1}\left(\mathbf{q}_{0}\right):=(0,0,1 ; 1,0,0), \\
& \mathbf{S}_{\Pi 3}\left(\mathbf{q}_{0}\right):=(0,1,0 ; 0,0,2), \\
& \mathbf{S}_{\Pi 4}\left(\mathbf{q}_{0}\right):=(1,0,0 ; 0,0,-1), \\
& \mathbf{S}_{\Lambda 2}\left(\mathbf{q}_{0}\right):=(0,0,1 ; 0,-2,0), \\
& \mathbf{S}_{7 \mathrm{R}}\left(\mathbf{q}_{0}\right):=\left(0,0,1 ; \frac{1}{2},-1,0\right),
\end{aligned}
$$

Define $\mathbf{x}_{i}:=\left(x_{i}^{1}, \ldots, x_{i}^{7}\right) \in \mathbb{R}^{7}$, where $x_{i}^{1}:=\mathrm{d}^{i} q_{\Pi 1} / \mathrm{d} t^{i}, x_{i}^{2}:=\mathrm{d}^{i} q_{\Pi 2} / \mathrm{d} t^{i}$, $x_{i}^{3}:=\mathrm{d}^{i} q_{\Lambda 1} / \mathrm{d} t^{i}, x_{i}^{4}:=\mathrm{d}^{i} q_{\Pi 3} / \mathrm{d} t^{i}, x_{i}^{5}:=\mathrm{d}^{i} q_{\Pi 4} / \mathrm{d} t^{i}, x_{i}^{6}:=\mathrm{d}^{i} q_{\Lambda 2} / \mathrm{d} t^{i}$ and $x_{i}^{7}:=$ $\mathrm{d}^{i} q_{7 \mathrm{R}} / \mathrm{d} t^{i}$. The solution set for the first order constraints is:

$$
\bar{K}_{\mathbf{q}_{0}}^{1}=K_{\mathbf{q}_{0}}^{1}=\left\{\left(x_{1}^{1}, 2 x_{1}^{1}, x_{1}^{3}, x_{1}^{1}, 2 x_{1}^{1}, x_{1}^{3},-2 x_{1}^{3}\right) \mid x_{1}^{1}, x_{1}^{3} \in \mathbb{R}\right\} .
$$

The solution set for the second order constraints is:

$$
\begin{aligned}
\bar{K}_{\mathbf{q}_{0}}^{2}=\left\{\left(\mathbf{x}_{1}, \mathbf{x}_{2}\right) \mid\right. & \mathbf{x}_{1}=\left(x_{1}^{1}, 2 x_{1}^{1}, 0, x_{1}^{1}, 2 x_{1}^{1}, 0,0\right), \\
\mathbf{x}_{2} & =\left(x_{2}^{1}, 2 x_{2}^{1}, x_{2}^{3}, x_{2}^{1}, 2 x_{2}^{1}, x_{2}^{3}, 4\left(x_{1}^{1}\right)^{2}-2 x_{2}^{3}\right), \\
& \left.x_{1}^{1}, x_{2}^{1}, x_{2}^{3} \in \mathbb{R}\right\},
\end{aligned}
$$

from which the second order approximation to the tangent cone is:

$$
K_{\mathbf{q}_{0}}^{2}=\pi_{1}\left(\bar{K}_{\mathbf{q}_{0}}^{2}\right)=\left\{\left(x_{1}^{1}, 2 x_{1}^{1}, 0, x_{1}^{1}, 2 x_{1}^{1}, 0,0\right) \mid x_{1}^{1} \in \mathbb{R}\right\}
$$

320 Since $\operatorname{dim}\left(K_{\mathbf{q}_{0}}^{2}\right)=1$ it is concluded that $\kappa_{1}=2$ and $C^{\mathrm{K}} V=K_{\mathbf{q}_{0}}^{2}$ as we know this is a 1-DOF mechanism. It can be seen that the tangent cone is only one vector space failing to reveal the bifurcation at $\mathbf{q}_{0}$. 
The solution set for the third order constraints is:

$$
\begin{aligned}
\bar{K}_{\mathbf{q}_{0}}^{3}= & \left\{\left(\mathbf{x}_{1}, \mathbf{x}_{2}, \mathbf{x}_{3}\right) \mid \mathbf{x}_{1} \in \pi_{1}\left(\bar{K}_{\mathbf{q}_{0}}^{2}\right), \mathbf{x}_{2} \in \pi_{2}\left(\bar{K}_{\mathbf{q}_{0}}^{2}\right),\right. \\
& \mathbf{x}_{3}=\left(x_{3}^{1}, 12\left(x_{1}^{1}\right)^{3}-12 x_{1}^{1} x_{2}^{3}+2 x_{3}^{1}, x_{3}^{3}, 12\left(x_{1}^{1}\right)^{3}-6 x_{1}^{1} x_{2}^{3}+x_{3}^{1}, 6\left(x_{1}^{1}\right)^{3}\right. \\
& \left.-9 x_{1}^{1} x_{2}^{3}+2 x_{3}^{1}, x_{3}^{3}, 12 x_{1}^{1} x_{2}^{1}-2 x_{3}^{3}\right), \\
& \left.x_{1}^{1}, x_{2}^{1}, x_{2}^{3}, x_{3}^{1}, x_{3}^{3} \in \mathbb{R}\right\},
\end{aligned}
$$

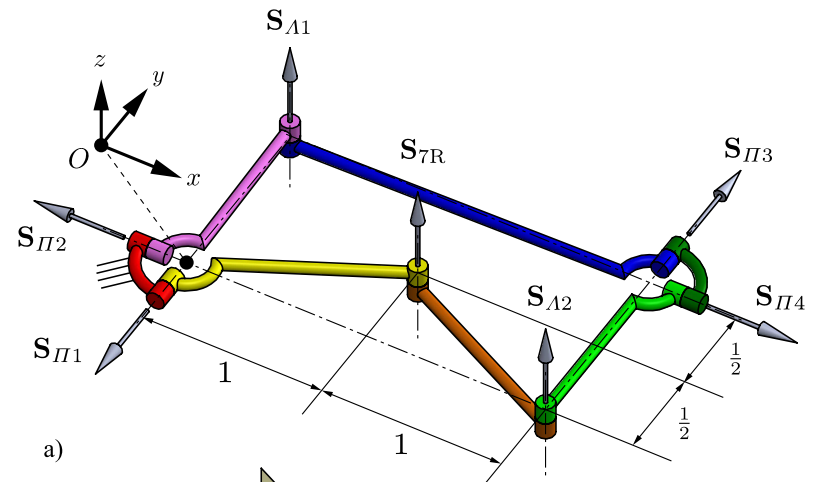

a)

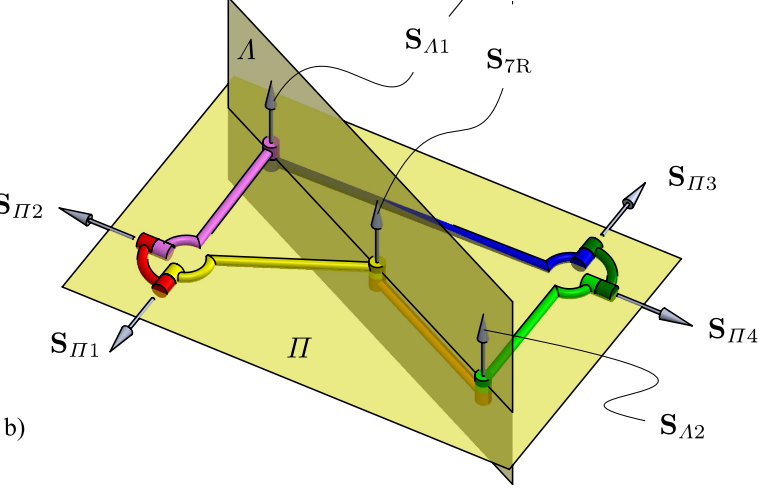

Figure 5: A line-symmetric-based 7R mechanism with a tangential intersection in the configuration space.

The solution set for the forth order constraints is:

$$
\bar{K}_{\mathbf{q}_{0}}^{4}=\bar{K}_{\mathbf{q}_{0}}^{4(\alpha)} \cup \bar{K}_{\mathbf{q}_{0}}^{4(\beta)}
$$


325

$$
\begin{aligned}
\bar{K}_{\mathbf{q}_{0}}^{4(\alpha)}= & \left\{\left(\mathbf{x}_{1}, \mathbf{x}_{2}, \mathbf{x}_{3}, \mathbf{x}_{4}\right) \mid \mathbf{x}_{1} \in \pi_{1}\left(\bar{K}_{\mathbf{q}_{0}}^{2}\right),\right. \\
& \mathbf{x}_{2}=\left(x_{2}^{1}, 2 x_{2}^{1}, 2\left(x_{1}^{1}\right)^{2}, x_{2}^{1}, 2 x_{2}^{1}, 2\left(x_{1}^{1}\right)^{2}, 0\right) \\
& \mathbf{x}_{3}=\left(x_{3}^{1},-12\left(x_{1}^{1}\right)^{3}+2 x_{3}^{1}, x_{3}^{3}, x_{3}^{1},-12\left(x_{1}^{1}\right)^{3}+2 x_{3}^{1}, x_{3}^{3}, 12 x_{1}^{1} x_{2}^{1}-2 x_{3}^{3}\right), \\
& \mathbf{x}_{4}=\left(x_{4}^{1}, 24\left(x_{1}^{1}\right)^{2} x_{2}^{1}-16 x_{1}^{1} x_{3}^{3}+2 x_{4}^{1}, x_{4}^{3}, 48\left(x_{1}^{1}\right)^{2} x_{2}^{1}-8 x_{1}^{1} x_{3}^{3}+x_{4}^{1},\right. \\
& \left.-12 x_{1}^{1} x_{3}^{3}+2 x_{4}^{1}, x_{4}^{3},-28\left(x_{1}^{1}\right)^{4}+12\left(x_{2}^{1}\right)^{2}+16 x_{1}^{1} x_{3}^{1}-2 x_{4}^{3}\right), \\
& \left.x_{1}^{1}, x_{2}^{1}, x_{3}^{1}, x_{3}^{3}, x_{4}^{1}, x_{4}^{3} \in \mathbb{R}\right\}
\end{aligned}
$$

and

$$
\begin{aligned}
\bar{K}_{\mathbf{q}_{0}}^{4(\beta)}= & \left\{\left(\mathbf{x}_{1}, \mathbf{x}_{2}, \mathbf{x}_{3}, \mathbf{x}_{4}\right) \mid \mathbf{x}_{1} \in \pi_{1}\left(\bar{K}_{\mathbf{q}_{0}}^{2}\right),\right. \\
& \mathbf{x}_{2}=\left(x_{2}^{1}, 2 x_{2}^{1},-\frac{6}{5}\left(x_{1}^{1}\right)^{2}, x_{2}^{1}, 2 x_{2}^{1},-\frac{6}{5}\left(x_{1}^{1}\right)^{2}, \frac{32}{5}\left(x_{1}^{1}\right)^{2}\right), \\
& \mathbf{x}_{3}=\left(x_{3}^{1}, \frac{132}{5}\left(x_{1}^{1}\right)^{3}+2 x_{3}^{1}, x_{3}^{3}, \frac{96}{5}\left(x_{1}^{1}\right)^{3}+x_{3}^{1}, \frac{84}{5}\left(x_{1}^{1}\right)^{3}+2 x_{3}^{1}, x_{3}^{3},\right. \\
& \left.12 x_{1}^{1} x_{2}^{1}-2 x_{3}^{3}\right), \\
& \mathbf{x}_{4}=\left(x_{4}^{1}, \frac{504}{5}\left(x_{1}^{1}\right)^{2} x_{2}^{1}-16 x_{1}^{1} x_{3}^{3}+2 x_{4}^{1}, x_{4}^{3}, \frac{432}{5}\left(x_{1}^{1}\right)^{2} x_{2}^{1}-8 x_{1}^{1} x_{3}^{3}+\right. \\
& x_{4}^{1}, \frac{288}{5}\left(x_{1}^{1}\right)^{2} a_{2}^{1}-12 x_{1}^{1} x_{3}^{3}+2 x_{4}^{1}, \frac{1632}{25}\left(x_{1}^{1}\right)^{4}+x_{4}^{3}, \frac{2948}{25}\left(x_{1}^{1}\right)^{4}+ \\
& \left.12\left(x_{2}^{1}\right)^{2}+16 x_{1}^{1} x_{3}^{1}-2 x_{4}^{3}\right), \\
& \left.x_{1}^{1}, x_{2}^{1}, x_{3}^{1}, x_{3}^{3}, x_{4}^{1}, x_{4}^{3} \in \mathbb{R}\right\}
\end{aligned}
$$

It can be seen that although $\pi_{1}\left(\bar{K}_{\mathbf{q}_{0}}^{4(\alpha)}\right)=\pi_{1}\left(\bar{K}_{\mathbf{q}_{0}}^{4(\beta)}\right), \pi_{2}\left(\bar{K}_{\mathbf{q}_{0}}^{4(\alpha)}\right) \neq$ $\pi_{2}\left(\bar{K}_{\mathbf{q}_{0}}^{4(\beta)}\right)$, therefore it is concluded that $\mathbf{q}_{0}$ is a tangential intersection of $V_{\alpha}$ and $V_{\beta}$. It is also concluded that $n_{C}=1$. Note that $\operatorname{dim}\left(\pi_{2}\left(\bar{K}_{\mathbf{q}_{0}}^{4(j)}\right)\right)=$ ${ }_{330} 2=2 \operatorname{dim}\left(\pi_{1}\left(\bar{K}_{\mathbf{q}_{0}}^{4(j)}\right)\right), j=\alpha, \beta$, therefore $\kappa_{2}=4$ and $\operatorname{dim}\left(\pi_{2}\left(\bar{K}_{\mathbf{q}_{0}}^{4(j)}\right)\right)$ will no longer decrease and the vector $\mathbf{x}_{2}$ will remain unchanged through the solution sets of any order. Observe that $\ddot{q}_{7 \mathrm{R}}\left(\mathbf{q}_{0}, \mathbf{x}_{1}\right)=x_{2}^{7}=0$ in branch $\alpha$ and $\ddot{q}_{7 \mathrm{R}}\left(\mathbf{q}_{0}, \mathbf{x}_{1}\right)=x_{2}^{7}=(32 / 5)\left(x_{1}^{1}\right)^{2} \neq 0$ in branch $\beta$, which proves that the seventh $\mathrm{R}$ joint is active in $\beta$. Figure 6 shows the $7 \mathrm{R}$ mechanism in two configurations 335 each belonging to each motion branch. 


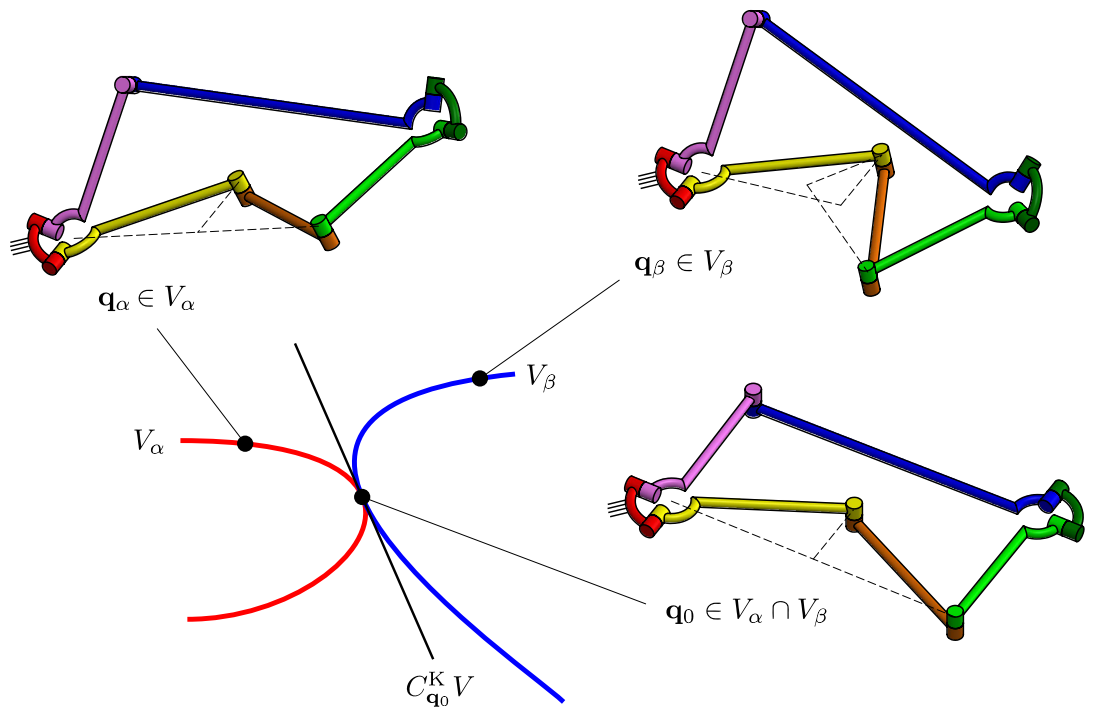

Figure 6: Branches of motion of the line-symmetric-based 7R mechanism.

\subsection{Case 2: A Schatz-based 7R mechanism}

Figure 7h shows a $7 \mathrm{R}$ mechanism in which joints with axes $\mathbf{S}_{\Pi 1}, \mathbf{S}_{\Pi 2} \mathbf{S}_{\Lambda 1}$, $\mathbf{S}_{\Pi 3}, \mathbf{S}_{\Lambda 2}$ and $\mathbf{S}_{\Pi 4}$ constitute a Schatz $6 \mathrm{R}$ linkage [63, 64]. As shown in figure 7b, in the configuration $\mathbf{q}_{0} \in V$, axes $\mathbf{S}_{\Pi 1}, \ldots, \mathbf{S}_{\Pi 4}$ lie on plane $\Pi$, while axes

$\mathbf{S}_{\Lambda 1}$ and $\mathbf{S}_{\Lambda 2}$ lie on $\Lambda$ and are perpendicular to $\Pi$. A seventh $\mathrm{R}$ joint is inserted between joints with axes $\mathbf{S}_{\Pi 3}$ and $\mathbf{S}_{\Lambda 2}$. At $\mathbf{q}_{0}$, the axis of the seventh joint, $\mathbf{S}_{7 \mathrm{R}}$, is parallel to $\mathbf{S}_{\Lambda 1}$ and also lies on $\Lambda$.

The screw coordinates with respect to the coordinate system with origin at $O$ shown in figure $7 \mathrm{~b}$ are the following:

$$
\begin{aligned}
\mathbf{S}_{\Pi 1}\left(\mathbf{q}_{0}\right) & :=(0,-1,0 ; 0,0,0), \\
\mathbf{S}_{\Pi 2}\left(\mathbf{q}_{0}\right) & :=(-1,0,0 ; 0,0,0), \\
\mathbf{S}_{\Lambda 1}\left(\mathbf{q}_{0}\right) & :=(0,0,1 ; 2,0,0), \\
\mathbf{S}_{\Pi 3}\left(\mathbf{q}_{0}\right) & :=\left(\frac{1}{2}, \frac{\sqrt{3}}{2}, 0 ; 0,0,1\right), \\
\mathbf{S}_{7 \mathrm{R}}\left(\mathbf{q}_{0}\right) & :=\left(0,0,1 ; \frac{1}{2},-\frac{3 \sqrt{3}}{2}, 0\right), \\
\mathbf{S}_{\Lambda 2}\left(\mathbf{q}_{0}\right) & :=(0,0,1 ; 0,-2 \sqrt{3}, 0), \\
\mathbf{S}_{\Pi 4}\left(\mathbf{q}_{0}\right) & :=(0,-1,0 ; 0,0,-2 \sqrt{3}),
\end{aligned}
$$

Define $\mathbf{x}_{i}:=\left(x_{i}^{1}, \ldots, x_{i}^{7}\right) \in \mathbb{R}^{7}$, where $x_{i}^{1}:=\mathrm{d}^{i} q_{\Pi 1} / \mathrm{d} t^{i}, x_{i}^{2}:=\mathrm{d}^{i} q_{\Pi 2} / \mathrm{d} t^{i}$, 
$x_{i}^{3}:=\mathrm{d}^{i} q_{\Lambda 1} / \mathrm{d} t^{i}, x_{i}^{4}:=\mathrm{d}^{i} q_{\Pi 3} / \mathrm{d} t^{i}, x_{i}^{5}:=\mathrm{d}^{i} q_{7 \mathrm{R}} / \mathrm{d} t^{i}, x_{i}^{6}:=\mathrm{d}^{i} q_{\Lambda 2} / \mathrm{d} t^{i}$ and $x_{i}^{7}:=$ $\mathrm{d}^{i} q_{\Pi 4} / \mathrm{d} t^{i}$. The solution set for the first order constraints is:
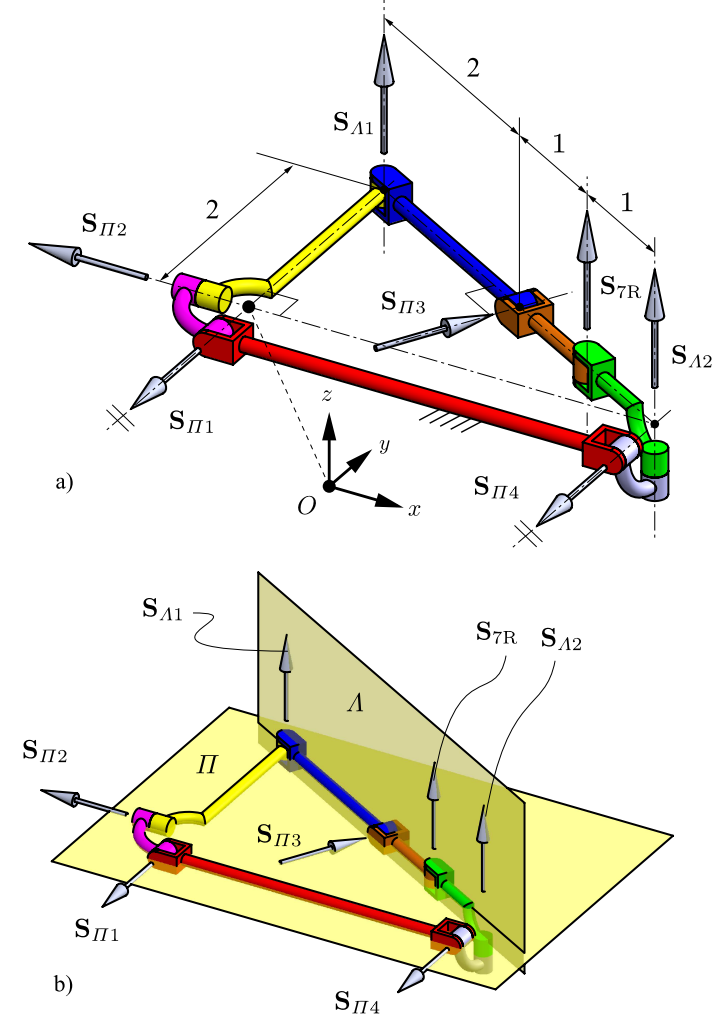

Figure 7: A Schatz-based 7R mechanism with a tangential intersection in the configuration space.

$$
\bar{K}_{\mathbf{q}_{0}}^{1}=K_{\mathbf{q}_{0}}^{1}=\left\{\left(x_{1}^{1}, \frac{\sqrt{3}}{2} x_{1}^{1},-\frac{1}{4} x_{1}^{5}, \sqrt{3} x_{1}^{1}, x_{1}^{5},-\frac{3}{4} x_{1}^{5}, \frac{1}{2} x_{1}^{1}\right) \mid x_{1}^{1}, x_{1}^{5} \in \mathbb{R}\right\} .
$$

The solution set for the second order constraints is:

$$
\begin{aligned}
\bar{K}_{\mathbf{q}_{0}}^{2}= & \left\{\left(\mathbf{x}_{1}, \mathbf{x}_{2}\right) \mid \mathbf{x}_{1}=\left(x_{1}^{1}, \frac{\sqrt{3}}{2} x_{1}^{1}, 0, \sqrt{3} x_{1}^{1}, 0,0, \frac{1}{2} x_{1}^{1}\right),\right. \\
& \mathbf{x}_{2}=\left(x_{2}^{1}, \frac{\sqrt{3}}{2} x_{2}^{1}, \frac{\sqrt{3}}{2}\left(x_{1}^{1}\right)^{2}-\frac{1}{4} x_{2}^{5}, \sqrt{3} x_{2}^{1}, x_{2}^{5}, \frac{\sqrt{3}}{4}\left(x_{1}^{1}\right)^{2}-\frac{3}{4} x_{2}^{5}, \frac{1}{2} x_{2}^{1}\right), \\
& \left.x_{1}^{1}, x_{2}^{1}, x_{2}^{5} \in \mathbb{R}\right\},
\end{aligned}
$$


from which the second order approximation to the tangent cone is:

$$
K_{\mathbf{q}_{0}}^{2}=\pi_{1}\left(\bar{K}_{\mathbf{q}_{0}}^{2}\right)=\left\{\left(x_{1}^{1}, \frac{\sqrt{3}}{2} x_{1}^{1}, 0, \sqrt{3} x_{1}^{1}, 0,0, \frac{1}{2} x_{1}^{1}\right) \mid x_{1}^{1} \in \mathbb{R}\right\}
$$

Since $\operatorname{dim}\left(K_{\mathbf{q}_{0}}^{2}\right)=1$ it is concluded that $\kappa=2$ and $C^{\mathrm{K}} V=K_{\mathbf{q}_{0}}^{2}$ as we know this is a 1-DOF mechanism. It can be seen that the tangent cone is only one vector space failing to reveal the bifurcation at $\mathbf{q}_{0}$.

The solution set for the third order constraints is:

$$
\begin{aligned}
\bar{K}_{\mathbf{q}_{0}}^{3}= & \left\{\left(\mathbf{x}_{1}, \mathbf{x}_{2}, \mathbf{x}_{3}\right) \mid \mathbf{x}_{1} \in \pi_{1}\left(\bar{K}_{\mathbf{q}_{0}}^{2}\right), \mathbf{x}_{2} \in \pi_{2}\left(\bar{K}_{\mathbf{q}_{0}}^{2}\right),\right. \\
& \mathbf{x}_{3}=\left(x_{3}^{1}, \frac{\sqrt{3}}{8}\left(4 x_{3}^{1}-\left(x_{1}^{1}\right)^{3}\right)+\frac{9}{8} x_{1}^{1} x_{2}^{5}, \frac{3 \sqrt{3}}{2} x_{1}^{1} x_{2}^{1}-\frac{1}{4} x_{3}^{5}, \frac{\sqrt{3}}{4}\left(4 x_{3}^{1}\right.\right. \\
& \left.\left.-\left(x_{1}^{1}\right)^{3}\right), x_{3}^{5}, \frac{3 \sqrt{3}}{4} x_{1}^{1} x_{2}^{1}-\frac{3}{4} x_{3}^{5},-\frac{3 \sqrt{3}}{8} x_{1}^{1} x_{2}^{5}+\frac{3}{4}\left(x_{1}^{1}\right)^{3}+\frac{1}{2} x_{3}^{1}\right), \\
& \left.x_{1}^{1}, x_{2}^{1}, x_{2}^{5}, x_{3}^{1}, x_{3}^{5} \in \mathbb{R}\right\},
\end{aligned}
$$

The solution set for the forth order constraints is:

$$
\bar{K}_{\mathbf{q}_{0}}^{4}=\bar{K}_{\mathbf{q}_{0}}^{4(\alpha)} \cup \bar{K}_{\mathbf{q}_{0}}^{4(\beta)}
$$

355

$$
\begin{aligned}
\bar{K}_{\mathbf{q}_{0}}^{4(\alpha)=} & \left\{\left(\mathbf{x}_{1}, \mathbf{x}_{2}, \mathbf{x}_{3}, \mathbf{x}_{4}\right) \mid \mathbf{x}_{1} \in \pi_{1}\left(\bar{K}_{\mathbf{q}_{0}}^{2}\right)\right. \\
& \mathbf{x}_{2}=\left(x_{2}^{1}, \frac{\sqrt{3}}{2} x_{2}^{1}, \frac{\sqrt{3}}{2}\left(x_{1}^{1}\right)^{2}, \sqrt{3} x_{2}^{1}, 0, \frac{\sqrt{3}}{4}\left(x_{1}^{1}\right)^{2}, \frac{1}{2} x_{2}^{1}\right) \\
& \mathbf{x}_{3}=\left(x_{3}^{1}, \frac{\sqrt{3}}{8}\left(4 x_{3}^{1}-\left(x_{1}^{1}\right)^{3}\right), \frac{3 \sqrt{3}}{2} x_{1}^{1} x_{2}^{1}-\frac{1}{4} x_{3}^{5}, \frac{\sqrt{3}}{4}\left(4 x_{3}^{1}-\left(x_{1}^{1}\right)^{3}\right),\right. \\
& \left.x_{3}^{5}, \frac{3 \sqrt{3}}{4} x_{1}^{1} x_{2}^{1}-\frac{3}{4} x_{3}^{5}, \frac{3}{4}\left(x_{1}^{1}\right)^{3}+\frac{1}{2} x_{3}^{1}\right), \\
& \mathbf{x}_{4}=\left(x_{4}^{1}, \frac{\sqrt{3}}{4}\left(2 x_{4}^{1}-3\left(x_{1}^{1}\right)^{2} x_{2}^{1}\right)+\frac{3}{2} x_{1}^{1} x_{3}^{5}, \frac{\sqrt{3}}{4}\left(7\left(x_{1}^{1}\right)^{4}+6\left(x_{2}^{1}\right)^{2}+\right.\right. \\
& \left.8 x_{1}^{1} x_{3}^{1}\right)-\frac{1}{4} x_{4}^{5},-\frac{\sqrt{3}}{2}\left(3\left(x_{1}^{1}\right)^{2} x_{2}^{1}-2 x_{4}^{1}\right), x_{4}^{5}, \frac{\sqrt{3}}{8}\left(7\left(x_{1}^{1}\right)^{4}+6\left(x_{2}^{1}\right)^{2}+\right. \\
& \left.\left.8 x_{1}^{1} x_{3}^{1}\right)-\frac{3}{4} x_{4}^{5},-\frac{\sqrt{3}}{2} x_{1}^{1} x_{3}^{5}+\frac{9}{2}\left(x_{1}^{1}\right)^{2} x_{2}^{1}+\frac{1}{2} x_{4}^{1}\right) \\
& \left.x_{1}^{1}, x_{2}^{1}, x_{3}^{1}, x_{3}^{5}, x_{4}^{1}, x_{4}^{5} \in \mathbb{R}\right\}
\end{aligned}
$$


and

$$
\begin{aligned}
\bar{K}_{\mathbf{q}_{0}}^{4(\beta)=} & \left\{\left(\mathbf{x}_{1}, \mathbf{x}_{2}, \mathbf{x}_{3}, \mathbf{x}_{4}\right) \mid \mathbf{x}_{1} \in \pi_{1}\left(\bar{K}_{\mathbf{q}_{0}}^{2}\right)\right. \\
& \mathbf{x}_{2}=\left(x_{2}^{1}, \frac{\sqrt{3}}{2} x_{2}^{1}, 0, \sqrt{3} x_{2}^{1}, 2 \sqrt{3}\left(x_{1}^{1}\right)^{2},-\frac{5 \sqrt{3}}{4}\left(x_{1}^{1}\right)^{2}, \frac{1}{2} x_{2}^{1}\right), \\
& \mathbf{x}_{3}=\left(x_{3}^{1}, \frac{\sqrt{3}}{8}\left(4 x_{3}^{1}+17\left(x_{1}^{1}\right)^{3}\right), \frac{3 \sqrt{3}}{2} x_{1}^{1} x_{2}^{1}-\frac{1}{4} x_{3}^{5}, \frac{\sqrt{3}}{4}\left(4 x_{3}^{1}-\left(x_{1}^{1}\right)^{3}\right),\right. \\
& \left.x_{3}^{5}, \frac{3 \sqrt{3}}{4} x_{1}^{1} x_{2}^{1}-\frac{3}{4} x_{3}^{5},-\frac{3}{2}\left(x_{1}^{1}\right)^{3}+\frac{1}{2} x_{3}^{1}\right), \\
& \mathbf{x}_{4}=\left(x_{4}^{1}, \frac{\sqrt{3}}{4}\left(15\left(x_{1}^{1}\right)^{2} x_{2}^{1}+2 x_{4}^{1}\right)+\frac{3}{2} x_{1}^{1} x_{3}^{5}, \frac{\sqrt{3}}{4}\left(16\left(x_{1}^{1}\right)^{4}+6\left(x_{2}^{1}\right)^{2}+\right.\right. \\
& \left.8 x_{1}^{1} x_{3}^{1}\right)-\frac{1}{4} x_{4}^{5},-\frac{\sqrt{3}}{2}\left(3\left(x_{1}^{1}\right)^{2} x_{2}^{1}-2 x_{4}^{1}\right), x_{4}^{5}, \frac{\sqrt{3}}{8}\left(25\left(x_{1}^{1}\right)^{4}+6\left(x_{2}^{1}\right)^{2}+\right. \\
& \left.\left.8 x_{1}^{1} x_{3}^{1}\right)-\frac{3}{4} x_{4}^{5},-\frac{\sqrt{3}}{2} x_{1}^{1} x_{3}^{5}+\frac{1}{2} x_{4}^{1}\right) \\
& \left.x_{1}^{1}, x_{2}^{1}, x_{3}^{1}, x_{3}^{5}, x_{4}^{1}, x_{4}^{5} \in \mathbb{R}\right\}
\end{aligned}
$$

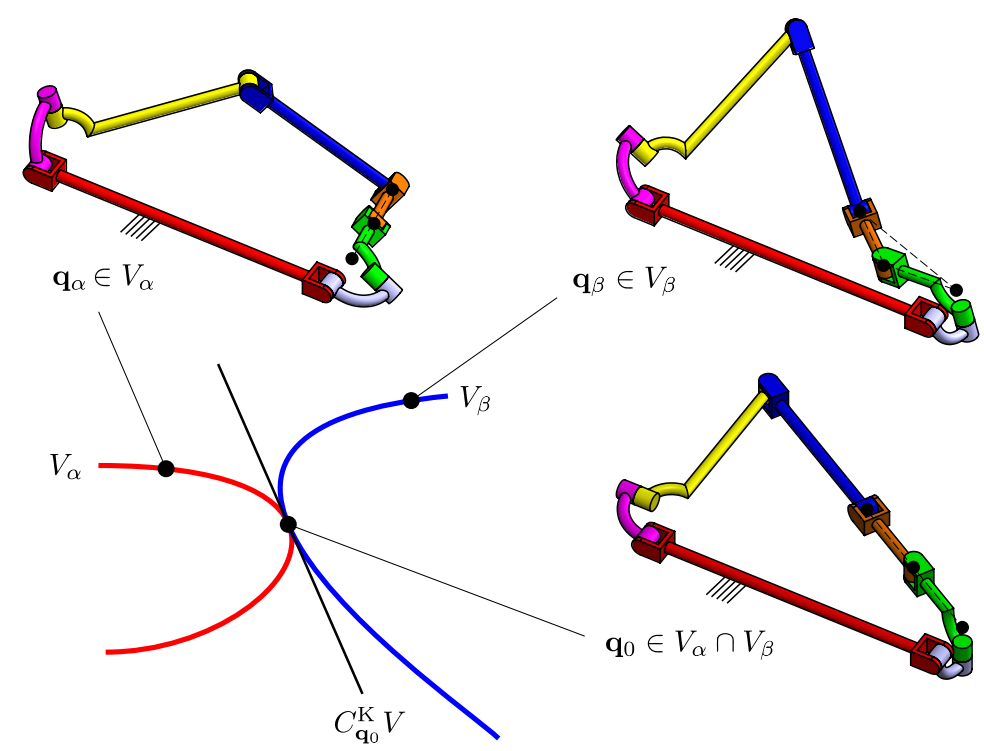

Figure 8: Branches of motion of the Schatz-based 7R mechanism.

It can be seen that $\pi_{1}\left(\bar{K}_{\mathbf{q}_{0}}^{4(\alpha)}\right)=\pi_{1}\left(\bar{K}_{\mathbf{q}_{0}}^{4(\beta)}\right)$ and $\pi_{2}\left(\bar{K}_{\mathbf{q}_{0}}^{4(\alpha)}\right) \neq \pi_{2}\left(\bar{K}_{\mathbf{q}_{0}}^{4(\beta)}\right)$, therefore $\mathbf{q}_{0}$ is a tangential intersection of $V_{\alpha}$ and $V_{\beta}$ with $n_{C}=1$. Since 
$\operatorname{dim}\left(\pi_{2}\left(\bar{K}_{\mathbf{q}_{0}}^{4(j)}\right)\right)=2=2 \operatorname{dim}\left(\pi_{1}\left(\bar{K}_{\mathbf{q}_{0}}^{4(j)}\right)\right), j=\alpha, \beta$, therefore $\kappa_{2}=4$. Ob-

serve that $\ddot{q}_{7 \mathrm{R}}\left(\mathbf{q}_{0}, \mathbf{x}_{1}\right)=x_{2}^{5}=0$ in branch $\alpha$ and $\ddot{q}_{7 \mathrm{R}}\left(\mathbf{q}_{0}, \mathbf{x}_{1}\right)=x_{2}^{5}=2 \sqrt{3}\left(x_{1}^{1}\right)^{2} \neq$ 0 in branch $\beta$, which proves that the seventh $\mathrm{R}$ joint is active in $\beta$. Figure 8 shows the $7 \mathrm{R}$ mechanism in two configurations each belonging to each motion branch, where geometric constraints lead to metamorphic behavior that limits the motion of links.

\section{Conclusions}

This paper presented the first examples of reconfigurable mechanisms whose configuration space contains tangent intersections of two branches of motion of the same dimension. It was pointed out that these singularities cannot be analyzed by computation of the kinematic tangent cone. In these examples, the 370 dimension of both branches is one. There is no theoretical restriction for designing mechanisms with this property and with mobility higher than one, however, this is out of the scope of the presented design method. A method for detecting branches of motion that intersect tangentially was also presented. This local method effectively detects the branches with common tangents at their intersection by considering solutions of the higher-order kinematic analyses, which are normally not considered in bifurcation analysis.

\section{Acknowledgement}

P.C. López-Custodio and Jian S. Dai thank the support of the Engineering and Physical Science Research Council (EPSRC) of the UK on grant numbers EP/P025447/1 and EP/P026087/1. P.C. López-Custodio thanks the Mexican National Council for Science and Technology (CONACyT) and the Advanced Kinematics and Reconfigurable Robotics Lab at Kings College for the support awarded to pursue doctoral studies. A. Müller acknowledges that this work has been supported by the "LCM K2 Center for Symbiotic Mechatronics" within the 385 framework of the Austrian COMET-K2 program. Xi Kang thanks the support of the National Natural Science Foundation of China (Grant No. 51535008, $51721003)$ and the International collaboration program (Grant No. B16034).

\section{References}

[1] D. Zlatanov, I. Bonev, C. Gosselin, Constraint singularities as configuration space singularities, in: J. Lenarčič, F. Thomas (Eds.), Advances in Robot Kinematics, Springer Netherlands, 2002, pp. 183-192.

[2] F. C. Park, J. W. Kim, Singularity analysis of closed kinematic chains, Journal of Mechanical Design 121 (1) (1999) 3238.

[3] K. H. Hunt, Kinematic Geometry of Mechanisms, Oxford University Press, New York, USA, 1978. 
[4] C. Gosselin, J. Ángeles, Singularity analysis of closed-loop kinematic chains, IEEE Transactions on Robotics and Automation 6 (3) (1990) 281-290.

[5] D. R. Walter, M. L. Husty, M. Pfurne, A complete kinematic analysis of the SNU 3-UPU parallel robot, in: D. J. Bates, G. M. Besana, S. D. Rocco, C. W. Wampler (Eds.), Interactions of Classical and Numerical Algebraic Geometry, Contemporary Mathematics, 2009, pp. 331-346.

[6] T. Arponen, S. Piipponen, J. Tuomela, Kinematical analysis of Wunderlich mechanism, Mechanism and Machine Theory 70 (2013) 16 - 31.

[7] L. Zhang, J. S. Dai, Reconfiguration of spatial metamorphic mechanisms, ASME Journal of Mechanisms and Robotics 1 (1) (2008) 011012 (8 pages).

[8] L. Zhang, D. Wang, J. S. Dai, Biological modeling and evolution based synthesis of metamorphic mechanisms, ASME Journal of Mechanical Design 130 (7) (2008) 072303 (11 pages).

[9] D. Gan, J. S. Dai, Q. Liao, Constraint analysis on mobility change of a novel metamorphic parallel mechanism, Mechanism and Machine Theory 45 (12) (2010) $1864-1876$.

[10] M. L. Husty, M. Pfurner, H.-P. Schrcker, K. Brunnthaler, Algebraic methods in mechanism analysis and synthesis, Robotica 25 (6) (2007) 661675.

[11] X. Kong, M. Pfurner, Type synthesis and reconfiguration analysis of a class of variable-dof single-loop mechanisms, Mechanism and Machine Theory 85 (2015) 116-128.

[12] J. Dai, J. R. Jones, Mobility in metamorphic mechanisms of foldable/erectable kinds, Transactions of the ASME: Journal of Mechanical Design 121 (3) (1999) 375-382.

[13] D. Gan, J. Dai, Q. Liao, Mobility change in two types of metamorphic parallel mechanisms, Transactions of the ASME Journal of Mechanisms and Robotics 1 (4) (2008) 9 pages.

[14] K. Zhang, J. Dai, Y. Fang, Topology and constraint analysis of phase change in the metamorphic chain and its evolved mechanism, ASME Journal of Mechanical Design 132 (12) (2010) 121001 (11 pages).

[15] J. Wei, J. S. Dai, Reconfiguration-aimed and manifold-operation based type synthesis of metamorphic parallel mechanisms with motion between $1 \mathrm{r} 2 \mathrm{t}$ and 2r1t, Mechanism and Machine Theory 139 (2019) 66 - 80.

[16] D. Gan, J. S. Dai, J. Dias, L. Seneviratne, Reconfigurability and unified kinematics modeling of a 3rtps metamorphic parallel mechanism with perpendicular constraint screws, Robotics and Computer-Integrated Manufacturing 29 (4) (2013) $121-128$. 
[17] K. Wohlhart, Kinematotropic linkages, in: J. Lenarčič, V. Parent-Castelli (Eds.), Recent Advances in Robot Kinematics, Portoroz, Slovenia. Dordrecht: The Netherlands, 1996, pp. 359-368.

[18] C. Galletti, P. Fanghella, Single-loop kinematotropic mechanisms, Mechanism and Machine Theory 36 (3) (2001) 743-761.

[19] G. Gogu, Branching singularities in kinematotropic parallel mechanisms, in: A. Kecskeméthy, A. Müller (Eds.), Computational Kinematics, Springer Berlin Heidelberg, Berlin, Heidelberg, 2009, pp. 341-348.

[20] P. C. López-Custodio, J. S. Dai, Design of a variable-mobility linkage using the Bohemian dome, in: International Design Engineering Technical Conferences and Computers and Information in Engineering Conference, Quebec, Canada, 2018.

[21] K. Zhang, J. S. Dai, Screw-system-variation enabled reconfiguration of the Bennett plano-spherical hybrid linkage and its evolved parallel mechanism, ASME Journal of Mechanical Design 137 (6) (2015) 10 pages.

[22] C. Lee, J. Hervé, Various types of RC//-like linkages and the discontinuously movable Koenigs joint, Mechanism and Machine Theory 96, Part 2 (2016) $255-268$.

[23] C.-C. Lee, J. M. Hervé, Discontinuously movable seven-link mechanisms via group-algebraic approach, Proceedings of the Institution of Mechanical Engineers, Part C: Journal of Mechanical Engineering Science 219 (6) (2005) 577-587.

[24] Q. Li, J. M. Hervé, Parallel mechanisms with bifurcation of Schoenflies motion, IEEE Transactions on Robotics 25 (1) (2009) 158-164.

[25] F. Aimedee, G. Gogu, J. Dai, C. Bouzgarrou, N. Bouton, Systematization of morphing in reconfigurable mechanisms, Mechanism and Machine Theory 96, Part 2 (2016) 215 - 224, reconfigurable mechanisms.

[26] P. C. López-Custodio, J. M. Rico, J. J. Cervantes-Sánchez, G. I. PérezSoto, Reconfigurable mechanisms from the intersection of surfaces, ASME Journal of Mechanisms and Robotics 8 (2) (2016) 021029-1—021029-19.

[27] X. Kong, Reconfiguration analysis of a 3-DOF parallel mechanism using Euler parameter quaternions and algebraic geometry method, Mechanism and Machine Theory 74 (2014) 188-201.

[28] S. Lu, D. Zlatanov, X. Ding, M. Zoppi, S. D. Guest, Reconfigurable chains of bifurcating type III Bricard linkages, in: X. Ding, X. Kong, J. S. Dai (Eds.), Advances in Reconfigurable Mechanisms and Robots II, Springer International Publishing, Cham, 2016, pp. 3-14. 
[41] C. Song, Y. Chen, I.-M. Chen, A 6R linkage reconfigurable between the line-symmetric Bricard linkage and the Bennett linkage, Mechanism and Machine Theory 70 (2013) $278-292$. 
[43] D. Gan, J. S. Dai, Geometry constraint and branch motion evolution of 3pup parallel mechanisms with bifurcated motion, Mechanism and Machine Theory 61 (2013) $168-183$.

[44] K. Zhang, J. S. Dai, Geometric constraints and motion branch variations for reconfiguration of single-loop linkages with mobility one, Mechanism and Machine Theory 106 (2016) $16-29$.

[45] X. Kong, Type synthesis of variable degrees-of-freedom parallel manipulators with both planar and 3T1R operation modes, in: Proceedings of the ASME 2012 International Design Engineering Technical Conferences,

[46] J. Wang, X. Kong, A novel method for constructing multi-mode deployable polyhedron mechanisms using symmetric spatial RRR compositional units, in: International Design Engineering Technical Conferences and Computers and Information in Engineering Conference, Quebec, Canada, 2018.

[53] P. C. López-Custodio, A. Müller, J. M. Rico, J. S. Dai, A synthesis method for 1-DOF mechanisms with a cusp in the configuration space, Mechanism and Machine Theory 132 (2019) $154-175$.

[54] A. Müller, Higher-order local analysis of kinematic singularities of lower pair linkages, in: Proceedings of the ASME 2017 International Design Engineering Technical Conferences and Computers and Information in Engineering Conference, Vol. 5B, Cleveland, Ohio, USA, 2017, paper number DETC2017-67039. 
[55] A. Müller, Local investigation of mobility and singularities of linkages, in: A. Müller, D. Zlatanov (Eds.), Singular Configurations of Mechanisms and Manipulators, Springer International Publishing, Cham, 2019, pp. 181-229.

[56] S. Piipponen, E. Hyry, T. Arponen, Kinematic analysis of multi-4-bar mechanisms using algebraic geometry, in: International Design Engineering Technical Conferences and Computers and Information in Engineering Conference, Cleveland, Ohio, USA, 2017.

[57] A. Müller, An overview of formulae for the higher-order kinematics of lowerpair chains with applications in robotics and mechanism theory, Mechanism and Machine Theory 142 (2019) 103594.

[58] P. C. López-Custodio, J. M. Rico, J. J. Cervantes-Sánchez, G. I. Pérez-Soto, C. R. Díez-Martínez, Verification of the higher order kinematic analyses equations, European Journal of Mechanics - A/Solids 61 (2017) 198 - 215.

[59] J. M. Rico, J. Gallardo, J. Duffy, Screw theory and the higher order kinematic analysis of serial and closed chains, Mechanism and Machine Theory 34 (4) (1999) 559-586.

[60] A. Müller, Local kinematic analysis of closed-loop linkages mobility, singularities, and shakiness, ASME Journal of Mechanisms and Robotics 8 (4) (2016) 041013-1-041013-11.

[61] J. E. Baker, An analysis of the Bricard linkages, Mechanisms and Machine Theory 15 (1980) 267-286.

[62] R. Bricard, Leçons de cinématique, Gauthier-Villars, Paris, France, 1927.

[63] P. Schatz, Rhytthmusforschung und Technik, Freies Geistesleben, Stuttgart, Germany, 1975.

[64] C.-C. Lee, J. S. Dai, Configuration analysis of the Schatz linkage, Proceedings of the Institution of Mechanical Engineers, Part C: Journal of Mechanical Engineering Science 217 (7) (2003) 779-786. 